\title{
Canine leishmaniasis prevalence in the Slovenian dog population
}

\author{
Tina Kotnik ${ }^{1 凶}$, Javier Moreno ${ }^{2}$, Barbara Šoba $^{3},{\text { Brane } \mathrm{Krt}^{4} \text {, Miha Skvarč }}^{5}$, \\ Aleksandra Vergles Rataj ${ }^{4}$, Maja Gorišek Bajc ${ }^{4}$, Urša Ravnik Verbič ${ }^{1}$ \\ ${ }^{1}$ Small Animal Clinic, Veterinary Faculty, University of Ljubljana, SI-1000 Ljubljana, Slovenia \\ ${ }^{2}$ WHO Collaborating Centre for Leishmaniasis Institute of Health Carlos III (ISCIII), Majadahonda, 228220 Madrid, Spain \\ ${ }^{3}$ Institute of Microbiology and Immunology, Faculty of Medicine, University of Ljubljana, SI-1000 Ljubljana, Slovenia \\ ${ }^{4}$ Institute of Microbiology and Parasitology, Veterinary Faculty, University of Ljubljana, SI-1000 Ljubljana, Slovenia \\ ${ }^{5}$ General Hospital Jesenice, Cesta maršala Tita 112, 4270 Jesenice, Slovenia \\ Tina.Kotnik@vf.uni-lj.si
}

Received: November 27, $2020 \quad$ Accepted: May 10, 2021

\begin{abstract}
Introduction: Leishmaniasis is a life-threatening zoonosis of which dogs are the major reservoir and sandflies are the vectors. Until now, the prevalence of canine leishmaniasis (CanL) in the Slovenian dog population was unknown. Material and Methods: Epidemiological data, eye swabs and blood samples were taken from 465 dogs born in Slovenia and older than one year. Commercial ELISA kits and real-time PCR were used. For ELISA-positive samples, an immunofluorescence antibody test (IFAT) was performed. Descriptive statistics were used to characterise the samples. The one-sample nonparametric chi-square test was used to test whether the categories of a variable were equally distributed. Results: A 59.9\% proportion of the recruited dogs had travelled to endemic regions and $62.1 \%$ of them had not been protected by insect repellents. Skin symptoms that might be CanL-related were described in 109 of the dogs' histories (23.4\%), inappetence and/or weight loss in 25 (5.4\%), and anaemia, intermittent fever, and/or lymphadenopathy in 19 (4.1\%). At the time of recruitment, all dogs were asymptomatic. All samples were PCR negative, nine (1.9\%) were ELISA positive, but none were IFAT positive. Five of the nine ELISA-positive dogs were non-travellers. Conclusion: We conclude that the seroprevalence of canine leishmaniasis of $1.9 \%$ in the autochthonous Slovenian dog population may pose a risk of endemic spread of the disease.
\end{abstract}

Keywords: canine leishmaniasis, CanL, prevalence, vector-borne diseases, dogs.

\section{Introduction}

The visceral form of human leishmaniasis (HumL) is a life-threatening disease that affects 200,000-400,000 people worldwide annually. It has the second highest mortality rate among parasitic diseases in humans with an estimated 20,000-40,000 deaths per year $(2,30)$. Leishmaniasis is also of great importance in veterinary medicine, as infection with Leishmania infantum can cause a severe, potentially fatal disease in dogs. If not diagnosed early, dogs lose weight heavily and exhibit intermittent fever, generalised lymphadenopathy, and exfoliative dermatitis. The most common cause of death or reason for euthanasia in affected dogs is renal failure (3). In addition, dogs are considered the main reservoir of these parasites for humans (12). Veterinarians must therefore aim to achieve two goals: first, to treat animal patients with canine leishmaniasis (CanL) in accordance with the latest treatment guidelines (30); and second, to prevent or minimise transmission from infected dogs to vectors and, consecutively, to other animals and humans.

Leishmaniasis is endemic in the Mediterranean region. Traditional endemic areas include Italy, Spain, Portugal, Greece, Bulgaria, Croatia and France (7), with Italy and Croatia bordering Slovenia. The endemic area has recently expanded towards northern Europe (27), and sandflies have recently been detected in regions previously mapped as free of the disease $(14,15,32)$. A high seroprevalence of CanL (31\%) was found in Dalmatia, a region of Croatia (35), while the overall seroprevalence in Croatia was recently reported to be $1.38 \%$ (22). Slovenia should not yet be considered an endemic region. Recently, updated data on HumL and CanL cases and the presence of competent Phlebotominae 
fly vectors in Slovenia have been reported. The same source also indicated an increase in the number of infected dogs that have been adopted in Slovenia (16). The presence of endemic areas in neighbouring countries (Italy and Croatia), an increased number of imported infected dogs and the simultaneous presence of competent Phlebotominae fly vectors in a climatically suitable region may lead to endemic spread of the disease. Therefore, the aim of the study was to determine the current prevalence of leishmaniasis among autochthonous dogs in Slovenia as a country with currently undetermined CanL status bordering endemic countries.

\section{Material and Methods}

As part of the study, a guide for the practical handling of leishmaniasis-infected dogs was prepared by the members of this project for practicing physicians in Slovenia. To inform dog owners, an article about leishmaniasis was published in a local newspaper with an invitation to participate in the study. The invitation was also published on the website of the Faculty of Veterinary Medicine of Ljubljana. Pet owners were informed by the participating veterinarians about the necessary preventive measures.

Dogs. Thirty private veterinary practices evenly distributed across the country were recruited to participate by collecting samples. Eye swabs and blood samples were collected from 465 dogs from April to October 2018, as statistically planned in advance according to a binomial equation. We considered the whole dog population to be 225884 deriving from the total of all registered dogs in Slovenia in February 2018 and $5 \%$ the response distribution (estimated based on precedents from other researchers) and decided to accept a $2 \%$ margin of error and tolerate a $95 \%$ confidence level. On this basis, 465 dogs were proportionately included from 10 regions (based on population data). There were two inclusion criteria: the dogs had to have been born in Slovenia and should have been at least one year old. Informed consent was provided by the dog owners for each sample, and a health status and travel history questionnaire was completed by each owner.

PCR. A real-time PCR was performed on eye swabs and blood using a genesig Leishmania Standard Kit (Primerdesign ${ }^{\mathrm{TM}}$ Ltd, Chandler's Ford, UK).

Serological analysis. Blood samples were tested using a commercial VetLine Leishmania ELISA kit (ref. LEIVT0310, Novatec, Dietzenbach, Germany), which uses native L. infantum antigens. For this analysis, promastigotes were grown on Roswell Park Memorial Institute/Serum Fetal Bovine (RPM/SFB) and a crude extract was harvested. As the ELISA test is semiquantitative, the results were expressed in units $(<9$ units - negative, 9-11 units - borderline, $>11$ units - positive). Blood samples from nine dogs that gave positive results with the ELISA kit were collected again in February 2019 and sent to the World Health Organization (WHO) Collaborating Centre for leishmaniasis, the Institute
Health Carlos III (ISCIII), Majadahonda, Madrid, Spain. They were tested by in-house indirect fluorescent antibody test (IFAT). Sera were tested in serial twofold dilutions from $1 / 10$ to $1 / 640$ which were applied to slides containing acetone-fixed $L$. infantum promastigotes and incubated at $37^{\circ} \mathrm{C}$ for $30 \mathrm{~min}$. After washing three times in phosphate-buffered saline, antibody fixation was revealed with fluorescein isothiocyanate-conjugated sheep anti-dog IgG (ICN, Aurora, OH, USA) diluted to $1 / 150$ in $0.01 \%$ Evans blue for counterstaining. Slides were incubated at $37^{\circ} \mathrm{C}$ for $30 \mathrm{~min}$, washed and examined with a fluorescence microscope. The titre corresponds to the final dilution at which at least $50 \%$ of the parasites have visible fluorescence. Dogs were considered infected when the titre was $\geq 1 / 160$.

Statistical analysis. All statistical tests were performed using SPSS Statistics 21 (IBM Corp., Armonk, NY, USA). Descriptive statistics were used to characterise the sample. The nonparametric one-sample chi-square test was used to test whether the categories of a variable were equally distributed, and a $\mathrm{P}$ value of $<0.05$ was considered statistically significant.

\section{Results}

Blood and eye swab samples were collected from 465 dogs of both sexes. The dogs' epidemiological data are presented in Table 1. Various breeds were represented among the dogs sampled (Table 2).

Table 1. Dogs' epidemiological data

\begin{tabular}{llcc}
\hline & & $\begin{array}{c}\text { Number } \\
\text { of dogs tested }\end{array}$ & $\begin{array}{c}\% \\
\text { of dogs tested }\end{array}$ \\
\hline Gender & & \\
\hline & male & 247 & 53.1 \\
\hline & female & 218 & 46.9 \\
\hline Age & & & \\
\hline & 1 to 3 years & 111 & 23.9 \\
\hline >3 to 7 years & 188 & 40.4 \\
\hline Travel & $\geq 8$ years & 166 & 35.7 \\
\hline outside Slovenia & 278 & 59.8 \\
\hline Insecticide use & & 23.0 \\
\hline repellents & 107 & 54.6 \\
\hline non-repellents & 254 & 8.2 \\
\hline both & 38 & 7.5 \\
\hline none & 35 & 6.7 \\
\hline undefined & 31 &
\end{tabular}

The median age of the dogs involved was 6 years (minimum 1 year, maximum 18 years), and the age distribution was $111(23.9 \%)$ 1-3 years old, $188>3-7$, and $166(35.7 \%) \geq 8$ years old. Almost two-thirds of the dogs $(59.8 \%)$ had travelled to endemic regions at least once in their lives. Many of them $(289 ; 62.1 \%$ of the recruits) were not protected with an insect repellent (Table 1). None of the included dogs were vaccinated against leishmaniasis. 
Owners were asked about any history of skin problems, anaemia, lymph node enlargement, recurrent high body temperature, anorexia, or weight loss in the dogs. The history of $126(27.1 \%)$ of the included dogs disclosed symptoms that could correspond to CanL (Table 3). The majority (109 dogs) had exhibited skin symptoms. Twenty-one (4.5\%) showed a combination of symptoms (Table 3). Three dogs had been diagnosed with leishmaniasis in the past, treated prior to inclusion, and had negative results in all tests at the time of the study. ELISA was performed on all 465 blood samples from the dogs. Nine (1.9\%) showed a positive ELISA result (Table 4). These dogs were re-sampled in February 2019 and additionally tested by IFAT, in which all 9 yielded a negative result. None of the 465 dogs included were PCR-positive by either eye swab test or blood test.

Table 2. Breeds of the recruited dogs

\begin{tabular}{lclclclc}
\hline Breed & Number & Breed & Number & Breed & Number & Breed & Number \\
\hline Mixed breed & 133 & Shih-tzu & 5 & Pug & 2 & Kerry blue terrier & 1 \\
\hline Golden retriever & 31 & Beagle & 5 & Jack Russell terrier & 2 & Chinese crested & 1 \\
\hline Labrador retriever & 22 & Newfoundland & 4 & Weimaraner & 2 & Short-haired dachshund & 1 \\
\hline German shepherd & 21 & $\begin{array}{l}\text { Australian } \\
\text { shepherd }\end{array}$ & 4 & Saint Bernard & 2 & Lagotto Romagnolo & 1 \\
\hline Boxer & 19 & Rottweiler & 4 & $\begin{array}{l}\text { Irish soft-coated wheaten } \\
\text { terrier }\end{array}$ & 2 & Toy poodle & 1 \\
\hline Border collie & 18 & Chihuahua & 4 & Caucasian shepherd & 2 & $\begin{array}{l}\text { German wirehaired } \\
\text { pointer }\end{array}$ & 1 \\
\hline German spaniel & 12 & Vizsla & 4 & Great Dane & 2 & Parson Russell terrier & 1 \\
\hline $\begin{array}{l}\text { American Staffordshire } \\
\text { terrier }\end{array}$ & 11 & Pekingese & 4 & Entlebucher & 2 & Pit bull terrier & 1 \\
\hline Cocker spaniel & 11 & Whippet & 4 & Schnauzer & 2 & $\begin{array}{l}\text { Montenegrin mountain } \\
\text { hound }\end{array}$ & 1 \\
\hline French bulldog & 9 & Airedale terrier & 3 & King Charles spaniel & 2 & Rhodesian ridgeback & 1 \\
\hline Bernese Mountain Dog & 8 & Beauceron & 3 & chow chow & 2 & White Swiss shepherd & 1 \\
\hline Alaskan Malamute & 7 & $\begin{array}{l}\text { Miniature } \\
\text { pinscher }\end{array}$ & 3 & Lakeland terrier & 1 & Welsh corgi & 1 \\
\hline West Highland terrier & 6 & Dobermann & 3 & Black Russian terrier & 1 & Hovawart & 1 \\
\hline Samoyed & 6 & Rough collie & 3 & Landseer & 1 & Havanese & 1 \\
\hline Tibetan terrier & 6 & Siberian husky & 3 & Maltese & 1 & Croatian Shepherd & 1 \\
\hline $\begin{array}{l}\text { Greater Swiss mountain } \\
\text { dog }\end{array}$ & 6 & Brandl-bracke & 3 & English springer spaniel & 1 & Irish red setter \\
\hline Yorkshire terrier & 5 & Malinois & 3 & Argentine mastiff & 1 & $\begin{array}{l}\text { Istrian coarse-haired } \\
\text { hound }\end{array}$ \\
\hline King Charles Cavalier & 5 & $\begin{array}{l}\text { Flat-coated } \\
\text { retriever }\end{array}$ & 3 & Bordeaux mastiff & 1 & Undefined breed & 1 \\
\hline Dalmatian & 5 & English bulldog & 2 & Coton de Tuléar & 1 & & Total \\
\hline Dachshund & 5 & Poodle & 2 & & &
\end{tabular}

Table 3. Disease symptoms commensurate with canine leishmaniasis from the history of dogs included in the study

\begin{tabular}{lcc}
\hline Past symptoms & Number & $\%$ \\
\hline None commensurate with canine leishmaniasis & 339 & 73.1 \\
symptoms that could correspond to CanL & 126 & 27.1 \\
a skin problems & 109 & 23.4 \\
banorexia or loss of weight & 25 & 5.4 \\
canaemia or lymph node enlargement or recurrent high body temperature & 19 & 4.1 \\
a \& b & 11 & 2.4 \\
b \& c & 3 & 0.6 \\
a \& c & 2 & 0.4 \\
a \& b \& c & 5 & 1.1 \\
\hline
\end{tabular}

Table 4. Dogs with positive ELISA results

\begin{tabular}{llllll}
\hline $\begin{array}{l}\text { Dog } \\
\text { identification } \\
\text { number }\end{array}$ & ELISA result & History of travelling & Ectoparasiticide use & $\begin{array}{l}\text { History of symptoms } \\
\text { corresponding to CanL }\end{array}$ & Breed \\
\hline 266 & $11.44 \mathrm{NTU}$ & None & Frontline & None & Mixed \\
\hline 265 & $12.37 \mathrm{NTU}$ & None & Frontline & None & German shepherd \\
\hline 225 & $11.60 \mathrm{NTU}$ & None & Nexgard & None & Caucasian shepherd \\
\hline 215 & $11.68 \mathrm{NTU}$ & None & No ectoparasiticide & None & Mixed \\
\hline 204 & $12.74 \mathrm{NTU}$ & SRB, CRO, A & Nexgard & None & German wirehaired pointer \\
\hline 191 & $13.71 \mathrm{NTU}$ & CRO & Foresto & Skin & Mixed \\
\hline 190 & $12.38 \mathrm{NTU}$ & CRO & Nexgard & None & Shih-tzu \\
\hline 186 & $13.44 \mathrm{NTU}$ & None & Frontline & Skin & German shepherd \\
\hline 172 & $22.30 \mathrm{NTU}$ & CRO, ITA, HUNG, A & Bravecto & None & Border collie \\
\hline
\end{tabular}

CanL - canine leishmaniasis; SRB - Serbia; CRO - Croatia; A - Austria; ITA - Italy; HUNG - Hungary 


\section{Discussion}

CanL affects approximately 2.5 million dogs annually in the Mediterranean region (21). Reports of seroprevalence in different countries in Europe vary and are highly dependent on the geographical and climatic conditions in a given part of the country. The average seroprevalence of CanL is $8.5 \%$ in Spain, $20.0 \%$ in Portugal and Cyprus, 4.0-20.0\% in Southern France, $2.0-15.0 \%$ in Italy, $25.0 \%$ in Greece, $15.7 \%$ in Turkey (7) and $1.4 \%$ in Croatia (22).

The sexes of the dogs involved were statistically equally distributed $\left(\chi^{2}=1.809, \mathrm{df}=1, \mathrm{P}=0.179\right)$. None of the included dogs were representatives of known resistant breeds (e.g. Ibizan dog) and 55 dogs (11.8\%) were representatives of susceptible breeds (21 German shepherds, 19 boxers, 11 cocker spaniels and 4 Rottweilers) $(6,10,28,29)$.

According to the literature, the distribution of the disease is bimodal with the highest prevalence in dogs younger than 3 years and older than 8 years (13). Our dogs were distributed by age as shown in Table 1, with $59.9 \%$ in either one or the other age range with the highest predilection to infection.

Preliminary studies showed that infected dogs were adopted from endemic regions and settled in Slovenia (16). Concern for the risk of spread of the disease from neighbouring endemic regions, as well as the possibility of endemic spread motivated the decision to conduct the present study. It was decided to include dogs older than one year and born in Slovenia. The reasons for this decision are explained as follows. It is known that a certain number of infected dogs and a certain number of competent vectors in an area are necessary for the endemic spread of the disease $(13,25)$. Unfortunately, entomological studies of phlebotomine vectors in the region are sparse (14). The criterion of including only dogs born in Slovenia was therefore set in order to reveal the endemic spread of the disease should positive reactors be found without any travel in their history. The second reason was to avoid the deliberate inclusion of sick dogs recently imported from endemic regions. During the preliminary studies, it became apparent that the collaborating veterinarians wanted to take advantage of free diagnostic evaluation for their clinical patients recently imported from Bosnia. This would bias the epidemiological study. We therefore decided to conduct the study in such a way as to enable us to find endemic infection using the history of the included dogs if they were positive in tests. In this case, it was decided that we would focus on dogs that had not travelled inbound to Slovenia.

At the time of the study, the dogs involved were clinically healthy. In the past, $27.1 \%$ of them had shown symptoms that could correspond to CanL (Table 3 ). The most common clinical manifestations of CanL include skin lesions with or without nail deformation (onychogryphosis), generalised lymphadenomegaly, loss of body weight, and intermittent fever. One of the most common laboratory abnormalities in CanL is nonregenerative anaemia $(3,30)$. We included these clinical signs in a questionnaire completed by the dog owners in our study. The observed symptoms were attributed to other diseases in all but three dogs. The medical history of these three dogs revealed that they had been previously diagnosed with CanL, and two of them had been treated. These three dogs had travelled to endemic regions (one to Italy, one to Croatia, and one to Italy, Austria, France, Switzerland, and the Czech Republic). At the time of our study, all three dogs were clinically in remission and showed antibody titres below the limit of detection.

The real-time PCR results of the eye swabs and blood in our study were all negative. The specificity of molecular tests is typically $95 \%$ to $100 \%$. One limitation is the sample source, which has a significant impact on the ability of molecular tests to identify infected dogs. Bone marrow and lymph node samples yield the highest number of positive results compared to other sites, regardless of clinical status (31). Nevertheless, collection of bone marrow and lymph node puncture biopsies is not suitable for epidemiological studies due to its invasive nature. Blood samples are easy to obtain but are not among the best tissues for PCR testing because the parasites are not constantly present in the bloodstream (30). In addition to blood samples, eye swabs were also collected in our study. According to current literature, they are considered good material for PCR $(8,17,31)$. In 2014, Ceccarelli et al. (5) showed that the sensitivity and specificity of PCR when testing conjunctival swabs were $87 \%$ and $96 \%$, respectively. Collection of eye swabs may in some cases have been from dogs carrying Leishmania but asymptomatic. This is a limitation to this study because it may have affected the total of negative PCR results; an underestimation of the presence of Leishmania may have resulted.

Many of the dogs involved in our study (59.8\%) had travelled to endemic regions at least once in their lives. Not all of them were sufficiently protected, either by effective repellents or by vaccination. Nevertheless, all but three remained healthy. This does not necessarily mean that they were not infected. We know that dogs can remain healthy if exposure to infected vectors is brief (e.g. during a vacation in an endemic area), if parasitic invasion is at low level, and if they are immunocompetent. The parasites may remain confined to the site of inoculation (skin) or to regional lymph nodes. We know that the majority of infected dogs never show symptoms (30).

Nine out of 465 blood samples gave low positive results with the ELISA test (Table 4). Our decision to perform an additional IFAT test was made on the basis that IFAT was considered a good standard test for CanL in non-endemic areas at the time of the study (1). However, considering current references, IFAT proves to be the best test only for symptomatic dogs and not for asymptomatic individuals. The sensitivity of IFAT in asymptomatic dogs may be only $29.4-31.25 \%(1,18)$. Because all dogs were asymptomatic at the time of 
recruitment, it is plausible that IFAT showed negative results in dogs with low positive titres determined by ELISA. In addition, sera tested with IFAT were collected later than those tested with ELISA. The collection was performed in February 2019. There is a high likelihood that the antibody titres of dogs that were positive by ELISA test in October 2018 fell below the threshold during the 2019 winter season. As shown in the study on levels of antibodies to phlebotomine salivary antigens, they are known to fluctuate seasonally. They rise in the spring and fall below detection levels in the winter. The canine humoral response to phlebotomine antigens correlates with the annual dynamics of sandfly activity expected for the region. Significantly lower IgG levels are observed during the non-transmission season (33). Since crossreactions and false positives must be considered in any serological test, ELISA is considered the most sensitive method in our case. The sensitivity and specificity of the ELISA used in our study were $95.8 \%$ and $95.43 \%$, respectively, according to the manufacturer's validation. The low titre positive results obtained in nine samples in our study should therefore correspond to infected but healthy dogs. According to the literature, low antibody levels are characteristic of subclinical infections or exposed but uninfected dogs (31). The majority of our ELISA positives ( 7 / 9) had no symptoms corresponding to CanL in their medical history. Five out of nine positives had never travelled abroad. Therefore, the infection or exposure must have occurred within Slovenia. Various routes of infection have been demonstrated in dogs (the placental route, through mating, through blood transfusion, and through infected sandfly bites) (30). Sandflies were recently detected in Slovenia by Ivović et al. (14), and to the authors' knowledge, that research is the only entomological study which has been conducted for this area. We do not know the actual occurrence or probable infectivity of sandflies in our country. However, the climate appears to be suitable as a sandfly habitat. Flies have also been detected in European countries north of Slovenia, such as Austria (26) and Germany (24). Detection of antisandfly salivary antibodies in the blood of dogs can be used as a marker of exposure to L. infantum vectors (33) and may help to distinguish between false-positive reactors and dogs with contact.

There are three main forms of human leishmaniasis: visceral, cutaneous, and mucocutaneous, ranging in severity from cutaneous lesions resolving without treatment through debilitating mucocutaneous infections to life-threatening visceral disease (4). Human visceral leishmaniasis is characterised by irregular episodes of fever, weight loss, hepatosplenomegaly and pancytopenia due to replication of Leishmania parasites in macrophages mainly in the liver, spleen and bone marrow (20). In the Mediterranean region, visceral leishmaniasis is caused by Leishmania infantum and is considered one of the major opportunistic infections in patients with HIV (20).
According to data in the literature, the prevalence of human leishmaniasis correlates directly with the number of infected dogs in a region (9). In the WHO European region, most (almost 75\%) of the human visceral leishmaniasis cases are reported from Albania, Georgia, Italy and Spain. Visceral leishmaniasis is endemic in nine countries of the European Union Bulgaria, Croatia, Cyprus, France, Greece, Italy, Malta, Portugal and Spain. Of these, Italy and Spain have the highest annual estimated incidence of 160-240 and 140-210 cases, respectively, followed by Greece with an annual estimated incidence of 50 to $80(2,11,34)$. In Croatia, the annual incidence is estimated to be 6-8 (2).

Recent reports of human leishmaniasis (HumL) cases in our country show low numbers. From 2010 to 2018, five cases of HumL were reported to the Slovenian National Institute of Public Health, including three cases of cutaneous leishmaniasis and two cases of the visceral infection. All of them were imported cases (23). The two patients with visceral leishmaniasis and one with the cutaneous type were infected in the Dalmatia region of neighbouring Croatia. Due to the low number of HumL cases, low seroprevalence in dogs in our country was expected. Nevertheless, global warming causing the northward spread of leishmaniasis vectors in Europe and importation of infected dogs into the country are two factors that increase the risk of establishing a new autochthonous transmission site for the parasite. The present work provides the basis for a national surveillance programme that should monitor the presence of canine and human cases of leishmaniasis in order to prevent the spread of the disease in Slovenia. Simultaneous concern for animal and human welfare is recommended by the World Health Organization in the One Health approach. Treatment of dogs may not completely eliminate parasites from the body, but it can significantly reduce the infectivity of treated dogs to vectors (19). Veterinarians must therefore treat canine patients with leishmaniasis in accordance with the latest treatment guidelines for the disease (30) and also educate dog owners about this threatening disease and its prevention measures.

We conclude that the leishmaniasis seroprevalence in autochthonous Slovenian dogs is $1.9 \%$. Five out of nine positive dogs had never travelled out of the country, which is a circumstance suggestive of the endemic spread of the disease. This should be confirmed in future studies.

Conflict of Interests Statement: The authors declare that there is no conflict of interests regarding the publication of this article.

Financial Disclosure Statement: World Health Organization provided financial support for this research. We gratefully acknowledge funding from the Slovenian Research Agency, program P4-0053 to T. Kotnik, program P4-0092 to B. Krt, A. Vergles Rataj 
and M. Gorišek Bajc, and program P3-0083 to B. Šoba and M. Skvarč. The funders had no influence on the study design, data collection and analysis, decision to publish, or preparation of the manuscript.

Animal Rights Statement: The project was reviewed and approved by the Ethics Committee of the Veterinary Faculty, University of Ljubljana. Informed consent from dog owners was provided for each specimen.

Acknowledgements: We would like to express our sincere gratitude to our colleague Javier Moreno and his laboratory staff for performing reference IFAT assays free of charge. We thank Dr. Petra Bandelj, PhD, for all efforts in isolating DNA from the eye swabs. For help with study design and statistical analysis of the data, we thank Mateja Nagode. For technical support, we thank technicians Edita Okršlar, Aleksander Jenko, Martina Germek, Tatjana Pačnik, Alenka Usenik, and Sabina Islamović. Last but not least, we would like to thank all dog owners for their participation and our veterinary colleagues in the field for sampling the dogs. We thank Prof. Patrick Bourdeau for inspiring some of us years ago that leishmaniasis has gained prominence in Europe and that we should draw public attention to this disease.

\section{References}

1. Adel A., Berkvens D., Abatih E., Soukehal A., Bianchini J., Saegerman C.: Evaluation of Immunofluorescence Antibody Test Used for the Diagnosis of Canine Leishmaniasis in the Mediterranean Basin: A Systematic Review and Meta-Analysis. PLoS One 2016, 11, e0161051, doi: 10.1371/journal.pone. 0161051.

2. Alvar J., Vélez I.D., Bern C., Herrero M., Desjeux P., Cano J., Jannin J., den Boer M.; WHO Leishmaniasis Control Team: Leishmaniasis worldwide and global estimates of its incidence. PLoS One 2012, 7, e35671, doi: 10.1371/journal.pone.0035671.

3. Bourdeau P., Saridomichelakis M.N., Oliveira A., Oliva G., Kotnik T., Gálvez R., Foglia Manzillo V., Koutinas A.F., da Fonseca I.P., Miró G.: Management of canine leishmaniosis in endemic SW European regions: a questionnaire-based multinational survey. Parasites \& Vectors 2014, 7, 110, doi: 10.1186/1756-3305-7-110. http://www.parasitesandvectors.com/ content $/ 7 / 1 / 110$.

4. Burza S., Croft S.L., Boelaert M.: Leishmaniasis. Lancet 2018, 392, 951-970, doi: 10.1016/S0140-6736(18)31204-2.

5. Ceccarelli M., Galluzzi L., Sisti D., Bianchi B., Magnani M.: Application of qPCR in conjunctival swab samples for the evaluation of canine leishmaniasis in borderline cases or disease relapse and correlation with clinical parameters. Parasit Vectors 2014, 7, 460, doi: 10.1186/s13071-014-0460-3.

6. De Vasconcelos T.C.B., Furtado M.C., Belo V.S., Morgado F.N., Figueiredo F.B.: Canine susceptibility to visceral leishmaniasis: a systematic review upon genetic aspects, considering breed factors and immunological concepts. Infect Genet Evol 2019, 74, 103293, doi: 10.1016/j.meegid.2017.10.005.

7. Dujardin J.C., Campino L., Cañavate C., Dedet J.P., Gradoni L., Soteriadou K., Mazeris A., Ozbel Y., Boelaert M.: Spread of Vector-borne Diseases and Neglect of Leishmaniasis, Europe. Emerg Infect Dis 2008, 14, 1013-1018, doi: 10.3201/ eid1407.071589.

8. Ferreira S. de A., Ituassu L.T., de Melo M.N., de Andrade A.S.: Evaluation of the conjunctival swab for canine visceral leishmaniasis diagnosis by PCR-hybridization in Minas Gerais State, Brazil. Vet Parasitol 2008, 152, 257-263, doi: 10.1016/j.vetpar.2007.12.022.

9. Ferroglio E., Battisti E., Zanet S., Bolla C., Concialdi E., Trisciuoglio A., Khalili S., Biglino A.: Epidemiological evaluation of Leishmania infantum zoonotic transmission risk in the recently established endemic area of Northwestern Italy. Zoonoses Public Health 2018, 65, 675-682, doi: 10.1111/zph.12477.

10. França-Silva J.C., da Costa R.T., Siqueira A.M., Machado-Coelho G.L., da Costa C.A., Mayrink W., Vieira E.P., Costa J.S., Genaro O., Nascimento E.: Epidemiology of canine visceral leishmaniosis in the endemic area of Montes Claros Municipality, Minas Gerais State, Brazil. Vet Parasitol 2003, 111, 161-173, doi: 10.1016/S0304-4017(02)00351-5.

11. Gradoni L.: Epidemiological surveillance of leishmaniasis in the European Union: operational and research challenges. Euro Surveill 2013, 18, 20539, doi: 10.2807/15607917.es2013.18.30.20539.

12. Gramiccia M., Gradoni L.: The current status of zoonotic leishmaniases and approaches to disease control. Int $\mathrm{J}$ Parasitol 2005, 35, 1169-1180, doi: 10.1016/j.ijpara.2005.07.001.

13. Harhay M.O., Olliaro P.L., Costa D.L., Costa C.H.: Urban parasitology: visceral leishmaniasis in Brazil. Trends Parasitol 2011, 27, 403-409, doi: 10.1016/j.pt.2011.04.001.

14. Ivović V., Kalan K., Zupan S., Bužan E.: Illegal waste sites as a potential micro foci of Mediterranean leishmaniasis: first records of phlebotomine sand flies (Diptera: psychodidae) from Slovenia. Acta Vet Beograd 2015, 65, 348-357, doi: 10.1515/acve-2015-0029.

15. Kasbari M., Ravel C., Harold N., Pesson B., Schaffner F., Depaquit J.: Possibility of Leishmaniasis Transmission in Jura, France. Emerg Infect Dis 2012, 18, 1030, doi: 10.3201/ eid1806.120158.

16. Kotnik T., Ivović V.: Living on the edge: border countries should have strict veterinary and health policy on leishmaniasis. In: The Epidemiology and Ecology of Leishmaniasis, edited by D. Claborn, Intech, Rijeka, 2017, doi: 10.5772/65273.

17. Leite R.S., Ferreira S. de A., Ituassu L.T., de Melo M.N., de Andrade A.S.: PCR diagnosis of visceral leishmaniasis in asymptomatic dogs using conjunctival swab samples. Vet Parasitol 2010, 170, 201-206, doi: 10.1016/j.vetpar.2010.02.020.

18. Mettler M., Grimm F., Capelli G., Camp H., Deplazes P.: Evaluation of enzyme-linked immunosorbent assays, an immunofluorescent-antibody test, and two rapid tests (immunochromatographic-dipstick and gel tests) for serological diagnosis of symptomatic and asymptomatic Leishmania infections in dogs. J Clin Microbiol 2005, 43, 5515-5519. doi: 10.1128/JCM.43.11.5515-5519.

19. Miró G., Gálvez R., Fraile C., Descalzo M.A., Molina R.: Infectivity to Phlebotomus perniciosus of dogs naturally parasitized with Leishmania infantum after different treatments. Parasit Vectors 2011, 4, 52, doi: 10.1186/1756-3305-4-52.

20. Monge-Maillo B., Norman F.F., Cruz I., Alvar J., López-Vélez R.: Visceral leishmaniasis and HIV coinfection in the Mediterranean region. PLoS Negl Trop Dis 2014, 8, e3021, doi: 10.1371/journal.pntd.0003021.

21. Moreno J., Alvar J.: Canine leishmaniasis: epidemiological risk and the experimental model. Trends Parasitol 2002, 18, 399-405, doi: 10.1016/S1471-4922(02)02347-4.

22. Mrljak V., Kuleš J., Mihaljević Ž., Torti M., Gotić J., Crnogaj M. Živičnjak T., Mayer I., Šmit I., Bhide M., Barić Rafaj R.: Prevalence and Geographic Distribution of Vector-Borne Pathogens in Apparently Healthy Dogs in Croatia. Vector Borne Zoonotic Dis 2017, 17, 398-408, doi: 10.1089/vbz.2016.1990.

23. National Institute of Public Health of the Republic of Slovenia. Epidemiološko spremljanje nalezljivih bolezni v Sloveniji. Leta 2010 2018 (Annual Epidemiological Report on Communicable Diseases in Slovenia. Years 2010-2018 - in Slovenian). National Institute of Public Health, Ljubljana, 2019. https://www.nijz.si/sl/epidemioloskospremljanje-nalezljivih-bolezni-letna-in-cetrtletna-porocila. 
24. Oerther S., Jöst H., Heitmann A., Lühken R., Krüger A., Steinhausen I., Brinker C., Lorentz S., Marx M., Schmidt-Chanasit J., Naucke T., Becker N.: Phlebotomine sand flies in Southwest Germany: an update with records in new locations. Parasites Vectors 2020, 13, 173, doi: 10.1186/s13071020-04058-6.

25. Özbel Y., Balcioğlu C., Kirami Ölgen M., Simsek F.M., Töz S.O., Ertabaklar H., Demir S., Alkan M.Z.: Spatial distribution of phlebotomine sand flies in the Aydin Mountains and surroundings: the main focus of cutaneous leishmaniasis in western Turkey. Journal Vector Ecol 2011, 36, 99-105, doi: 10.1111/j.1948-7134.2011.00118.x

26. Poeppl W., Obwaller A.G., Weiler M., Burgmann H., Mooseder G., Lorentz S., Rauchenwald F., Aspöck H., Walochnik J., Naucke T.J.: Emergence of sandflies (Phlebotominae) in Austria, a Central European country. Parasitol Res 2013, 112, 4231-4237, doi: 10.1007/s00436-013-3615-9.

27. Ready P.D.: Leishmaniasis emergence in Europe. Euro Surveill 2010, 15, 19505.

28. Sideris V., Papadopoulou G., Dotsika E., Karagouni E.: Asymptomatic canine leishmaniasis in Greater Athens area, Greece. Eur J Epidemiol 1999, 15, 271-276, doi: 10.1023/A:1007526401175.

29. Solano-Gallego L., Llull J., Ramos G., Riera C., Arboix M., Alberola J., Ferrer L.: The Ibizian hound presents a predominantly cellular immune response against natural Leishmania infection. Vet Parasitol 2000, 90, 37-45, doi: 10.1016/S03044017(00)00223-5.
30. Solano-Gallego L., Miró G., Koutinas A., Cardoso L., Pennisi M.G., Ferrer L., Bourdeau P., Oliva G., Baneth G.: LeishVet guidelines for the practical management of canine leishmaniosis. Parasit Vectors 2011, 4, doi: 10.1186/1756-3305-4-86.

31. Travi B.L., Cordeiro-da-Silva A., Dantas-Torres F., Miró G.: Canine visceral leishmaniasis: Diagnosis and management of the reservoir living among us. PLoS Negl Trop Dis 2018, 12, e0006082, doi: 10.1371/journal. pntd.0006082.

32. Vaselek S., Ayhan N., Oguz G., Erisoz Kasap O., Savić S., Di Muccio T., Gradoni L., Ozbel Y., Alten B., Petrić D.: Sand fly and Leishmania spp. survey in Vojvodina (Serbia): first detection of Leishmania infantum DNA in sand flies and the first record of Phlebotomus (Transphlebotomus) mascittii Grassi, 1908. Parasites Vectors 2017, 10, 444, doi: 10.1186/s13071-017-2386-z.

33. Velez R., Spitzova T., Domenech E., Willen L., Cairó J., Volf P., Gállego M.: Seasonal dynamics of canine antibody response to Phlebotomus perniciosus saliva in an endemic area of Leishmania infantum. Parasites Vectors 2018, 11, 545, doi: 10.1186/s13071018-3123-y.

34. World Health Organization Regional Office for Europe, Gradoni L., López-Vélez R., Mokni M.: Manual on case management and surveillance of the leishmaniases in the WHO European Region. WHO Regional Office for Europe Publications, Copenhagen, 2017. https://www.euro.who.int/ data/assets/pdf file/0006/ 341970/MANUAL-ON-CASE-MANAGEMENT_FINAL_withcover-and-ISBN.pdf.

35. Živičnjak T., Martinković F., Khoury C., Bongiorno G., Bosnić S., Lukačević D., Maroli M.: Serological and entomological studies of canine leishmaniosis in Croatia. Vet Arch 2011, 81, 99-110. 